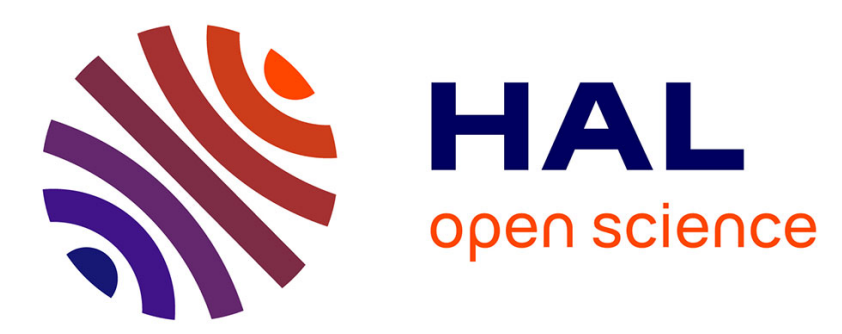

\title{
Depth-dependent chemical and magnetic local orders in thin magnetic films
}

Narcizo M. Souza-Neto, Aline Y. Ramos, Hélio C. N. Tolentino, Alessandro Martins, Antonio D. Santos

\section{- To cite this version:}

Narcizo M. Souza-Neto, Aline Y. Ramos, Hélio C. N. Tolentino, Alessandro Martins, Antonio D. Santos. Depth-dependent chemical and magnetic local orders in thin magnetic films. Applied Physics Letters, 2006, 89, pp.111910. 10.1063/1.2335782 . hal-00115980

\author{
HAL Id: hal-00115980 \\ https://hal.science/hal-00115980
}

Submitted on 24 Nov 2006

HAL is a multi-disciplinary open access archive for the deposit and dissemination of scientific research documents, whether they are published or not. The documents may come from teaching and research institutions in France or abroad, or from public or private research centers.
L'archive ouverte pluridisciplinaire HAL, est destinée au dépôt et à la diffusion de documents scientifiques de niveau recherche, publiés ou non, émanant des établissements d'enseignement et de recherche français ou étrangers, des laboratoires publics ou privés. 


\title{
Depth-dependent chemical and magnetic local order in thin magnetic films
}

\author{
Narcizo M. Souza-Neto \\ Laboratório Nacional de Luz. Síncrotron, CP 6192, 13084-971, Campinas, Brazil and \\ Departamento de Física dos Materiais e Mecânica, Instituto de Física, Universidade de São Paulo, São Paulo, Brazil
}

Aline Y. Ramos

Laboratoire Louis Néel, CNRS-UPR 5051, Grenoble, France and

Laboratório Nacional de Luz Síncrotron, CP 6192, 13084-971, Campinas, Brazil

Hélio C. N. Tolentino

Laboratoire de Cristallographie, CNRS-UPR 5031, Grenoble, France and

Laboratório Nacional de Luz Síncrotron, CP 6192, 13084-971, Campinas, Brazil

\author{
Alessandro Martins \\ Instituto de Física, Universidade Federal de Goiás, Goiânia, Brazil
}

Antonio D. Santos

Departamento de Física dos Materiais e Mecânica, Instituto de Física, Universidade de São Paulo, São Paulo, Brazil

(Dated: 24th November 2006)

\begin{abstract}
We report on the use of $\mathrm{x}$-ray absorption spectroscopy with resolved grazing incidence to clarify the thicknessdependent magnetic properties in nanometric CoPt films. We show that in the thinnest samples the chemical order that induces the perpendicular magnetic anisotropy has no depth dependence. However in the thicker samples the chemical order is depth-dependent along the film thickness, with a disordered layer close to the substrate. The ability of the experimental approach to adress the depth-dependence of the local structural parameters make it an unique tool, suitable for a large variety of nanometric structure where this dependence is an important problem.
\end{abstract}

Improvement of low-cost high-storage density magnetic media is an emerging technological challenge. It requires a substantial understanding of the complex relationship between thickness and physical properties, often dominated by microstructural effects localized at the interfaces between magnetic and metal layers. Such effects can only be studied using experimental techniques able to peer selectively along the sample's depth. Since at grazing incidence the sampling depth of the x-rays strongly depends on the incidence angle the structural issue can be adressed by combining established $\mathrm{X}$-ray techniques to grazing incidence setups. Analysis techniques based on X-ray diffraction (XRD) and giving the long range order as a function of the probed thickness have been successfully developped in the last past decades [1-3]. X-ray Absorption Spectroscopy (XAS) is widely known as element selective probe of the local atomic and electronic structure [4]. The versatility in the geometries and detection schemes enables its association to advanced experimental setups, providing renewed original insights to the material science. However, even if grazing angle XAS is already used worldwide, its sensitivity in probing in-depth local order is still an unexplored issue.

Under specific growth conditions, the equiatomic FePt and CoPt nanometric films develop a chemical ordering along the $\mathrm{c}$ axis ( $\mathrm{L} 1_{0}$ tetragonal phase) in the normal film direction, giving rise to perpendicular magnetic anisotropy (PMA) [5, 6]. Many structural parameters are determinants for the films quality. Fine characterization of the stoichiometry and the structural order is mandatory, as well as a proper determination of the surface states $[7,8]$. Depth heterogeneity may also deeply af- fect the overall performance of the films. Moreover, it shoud be kept in mind that anisotropic chemical order at short range scale is sufficient to promote PMA $[9,10]$. A crucial issue is then to fully recover the 3D-information, as well at the short range as at the long range scale [11]. We report here on the depth local information obtained in CoPt films by combining XAS along with resolved grazing angle setup.

CoPt films were prepared by magnetron sputtering, following an experimental procedure promoting the growth of the $\mathrm{L} 1_{0}$ chemically ordered phase normally to the film surface [12]. The samples were grown by combined deposition from the pure targets of elemental $\mathrm{Co}$ and $\mathrm{Pt}$ onto singlecrystal $\mathrm{MgO}(100)$ hot substrates $\left(500^{\circ} \mathrm{C}\right)$ covered with a $\mathrm{Pt}$ $50 \mathrm{~nm}$ thick fcc(100) buffer. The deposition rate was approximately $1 \AA / s$. The chamber base pressure was on the order of $1 \times 10^{-6} \mathrm{~Pa}$ and the sputtering ambient was $0.6 \mathrm{~Pa}$ high purity Ar. The films were capped with a $2 \mathrm{~nm}$ thick Pt layer to protect them from oxidation. XRD pole figures around the main reflection beams established the epitaxial quality. The films roughness measured by Atomic Force Microscopy was less than $1 \mathrm{~nm}$. The nominal thicknesses of the samples are 10nm (CoPt10), 30nm (CoPt30), 50nm (CoPt50) and 200nm (CoPt200).

The magnetic properties of the films exhibit a singular thickness dependence (figure 1). The overall PMA is well defined in the thinnest sample (CoPt10), largely lost in the samples with intermediate thickness (CoPt30 and CoPt50) and partially recovered in the thickest sample (CoPt200). The thickness dependence is well correlated to the variations in the long range order parameter $\mathrm{S}$ of the $\mathrm{L} 1_{0}$ phase $[5,12]$ obtained 
by XRD. The CoPt10 has a good long range order $(S=0.47)$, CoPt30 and CoPt50 are almost fully disordered $(S=0.02)$, and CoPt200 shows a limited long range order $(S=0.19)$. The narrow relationship between the structural order and the PMA is then clear. The scope of this study is to elucidate more precisely whether the lowering of the PMA performances are due to homogeneous disorder or to in-depth structural heterogeneity within the thicker films.

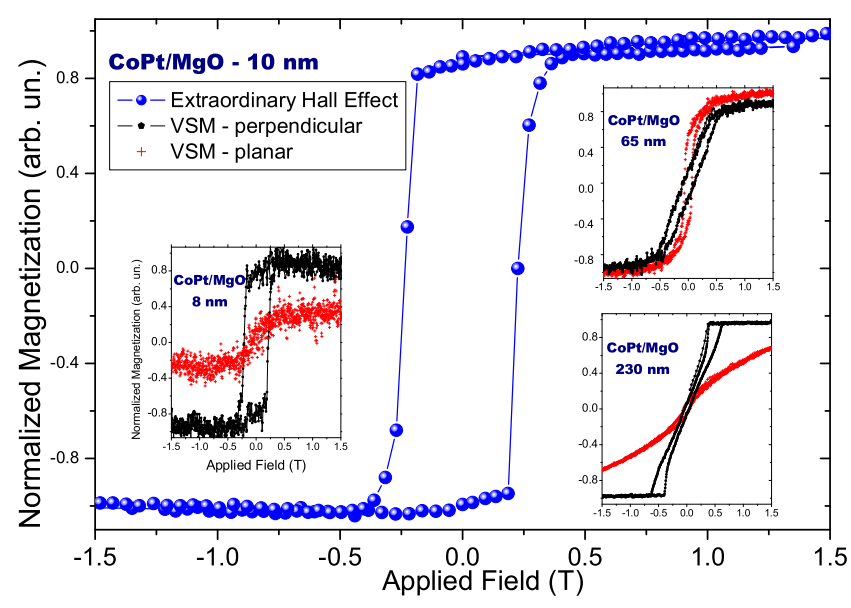

Figure 1: Magnetic hysteresis loops for CoPt films. The main graph shows the loop obtained by Extraordinary Hall Effect for CoPt10, with the magnetic field perpendicular to the film surface. The insets present the measurements using a Vibrating Sample Magnetometer for CoPt10, CoPt50 and CoPt200, with the external magnetic field planar and perpendicular to the film surface. The data were normalized by the film volume.

In metals and alloys, $x$-ray beams have an attenuation length $\lambda$ of several micrometers. However, at grazing incidence conditions, i.e. below the critical angle for total reflection $\theta_{C}$, standing waves at the surface of the material limit the beams to evanescent waves confined within a few nanometers below the reflecting surface of the material $[13,14] . \theta_{C}$ and $\lambda$ are related through the Fresnel equations and their exact dependence can be calculated by standard methods for specular reflection [14]. Close to $\theta_{C}$, small changes in the grazing angle induce large variations in the attenuation length. For CoPt alloys, around $\theta_{C}\left(\approx 0.51^{\circ}\right.$ at the Co K-edge $)$, a typical step of $\approx+0.01^{\circ}$ corresponds to an increase of the attenuation length by about $1 \mathrm{~nm}$. When XAS is performed at grazing incidence, the penetration depth at the energy $\mathrm{E}$ is known with such nanometric resolution. The XAS spectra correspond to the integration, $\int_{0}^{t} \mu(E ; z) d z$, of the contributions of infinitesimal sheets with thicknesses $d z$ at the depth $z$ from sample surface. The collection of the spectra at various grazing angles directly informs on the in-depth homogeneity of the local structure.

The grazing incidence $\mathrm{x}$-ray absorption measurements were performed at the LNLS. The setup includes $20 \mu \mathrm{m}$-vertical slits limiting the beam size on the sample mounted on a high precision goniometer. XANES (X-ray Absorption Near Edge Structure) spectra were collected in the fluorescence mode at the D04B-XAFS1 beamline [15] with a Si (111) channel-cut

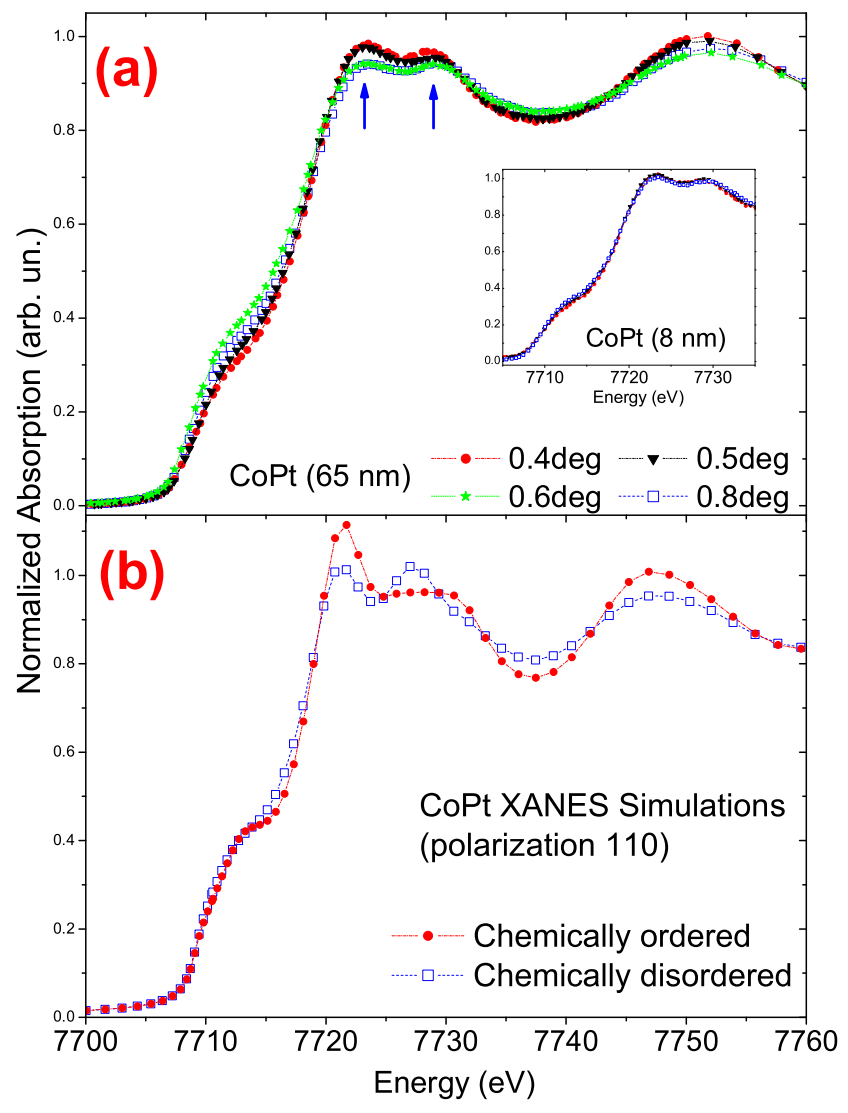

Figure 2: (a) XANES experimental data at some typical grazing angles for CoPt50. The lower inset shows the same spectra for CoPt10. (b) XANES ab initio simulations of chemically ordered and disordered CoPt clusters for in-plane polarization.

monochromator. The incident beam intensity and the energy calibration were monitored using a ion-chamber and a Co metal standart. The reflected beam and the Co fluorescence emission were collected using a second ion-chamber and a 15 element Ge detector, respectively. The fluorescence emission and/or x-ray reflectivity curves were used to calibrate and select with an accuracy of $\approx 0.01^{\circ}$ the working grazing angle corresponding to a chosen penetration depth.

For the thinnest sample CoPt10, the shape and relative intensity of the two scattering resonances above the edge are unchanged in the XANES data collected at several grazing incidence (figure $2 \mathrm{a}$, inset). This result demonstrates the homogeneity of the Co environment across the film. For the other samples, the relative intensity of the first main resonance steadily decreases, in relation to the second one, above a given grazing incidence (figure $2 \mathrm{a}$ ). The drop occurs close to $\theta_{C}$, but at slightly larger value for CoPt200 than for CoPt30 and CoPt50, i.e. for larger penetration depth.

$\mathrm{Ab}$ initio simulations [16] of XANES spectra in grazing incidence geometry have been performed on model structures of chemically ordered and disordered clusters (figure $2 b$ ). They show that the relative intensities of the two main resonances are related to the chemical order, the first resonance being more intense for the chemically ordered structure. The ex- 
perimental data account then for a loss of the local chemical order around the Co atoms, above a given incident angle. The experimental value of this angle is used to obtain an evaluation of the disordered layer thickness [14] which is around $20 \mathrm{~nm}$ for CoPt30 and CoPt50, and 40nm for CoPt200. The overall magnetic behavior of each sample is then determined by the ratio between the thickness of the disordered layer and the total CoPt film thickness. For CoPt30 and CoPt50 this ratio is around 0.5 and the PMA is lost. For CoPt200 the ratio is about 0.2 and a significant PMA component is kept.

To further confirm this statement, we associated X-ray Resonant Magnetic Scattering (XRMS) to the grazing angle setup [17]. The data were collected at the Co K-edge at the D06BDXAS LNLS beamline [18]. Circularly polarised x-rays were selected about $0.3 \mathrm{mrad}$ above the orbite plane and the magnetic contrast was obtained by flipping a magnetic field of 0.45Tesla, applied along the beam direction in the film's surface. The contrast in the scattering -asymmetry ratio- probes the local magnetic properties and informs, in the geometry of the experiments, on the in-plane local magnetic moment carried by the Co atoms. The measure of the asymmetry ratio at different grazing angles provides information on the depth dependence of this local moment. Figure 3 displays the curves obtained for a $200 \mathrm{~nm}$ thick sample. At grazing angles below $\theta_{C}$, the amplitude of the XRMS signal is mainly dominated by the experimental noise. There is no magnetic contrast because the $\mathrm{x}$-rays are probing a slab of sample where the local Co magnetic moment has no in plane component. When $\theta$ increases above $\theta_{C}$ a small contribution of in plane magnetic moment is observed, confirming the existence of the disordered layer close to the interface with the buffer.

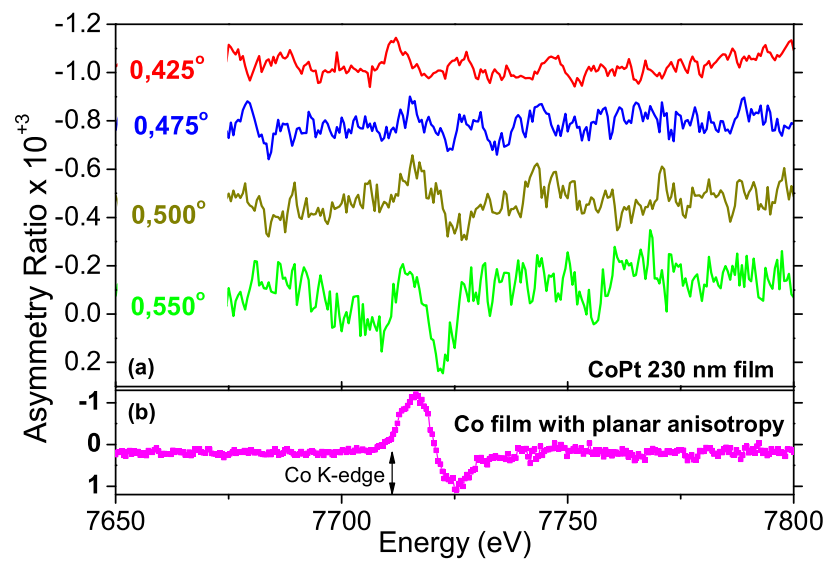

Figure 3: (a) Asymmetry ratio in XRMS, for a $200 \mathrm{~nm}$-thick film and several incident grazing angles. The different spectra are shifted for clarity. A reference XRMS spectrum obtained for a $50 \mathrm{~nm}$-thick Co film with total planar magnetic anisotropy is shown for comparison in (b).

Angle resolved grazing incidence XAS and XRMS succeed in directly correlate the modifications of the average magnetic performances of the films to an in-depth drop in the chemical order. While the thin CoPt10 films is depth-homogeneous, thicker films, grown under identical conditions, develop a chemically disordered layer at the interface with the buffer. The thickness of the disorder layer seems almost independent (around $\approx 20 \mathrm{~nm}$ ) on the total thicknesses. More precise determination of this thickness, as well as a discussion of the roughness of the order-disorder interface require further analytical tools, actually in development. The above results demonstrate the strong potentiality of the experimental approach, appropriate to clarify the local organisation in nanometric structures where the depth dependence of the local parameters cannot be ruled out.

The authors are indebted to Laurent Ranno and Baptiste Carvello for Extraordinary Hall Effect and to Marcia Fantini for XRD. Thanks to Flávio Garcia and Márcio Medeiros for providing the Co standart for XRMS experiments. This work is supported by LNLS/ABTLuS/MCT and by the Brazilian agencies CNPq, SECTEC/GO and FAPESP.

[1] T. C. Q. Noakes, P. Bailey, P. K. Hucknall, K. Donovan, and M. A. Howson, Phys. Rev. B 58, 4934 (1998).

[2] H. Dosch, Phys. Rev. B 43, 13172 (1991).

[3] M. D. Santis, R. Baudoing-Savois, P. Dolle, and M. C. SaintLager, Phys. Rev. B 66, 085412 (2002).

[4] D. E. Sayers, E. A. Stern, and F. W. Lytle, Phys. Rev. Lett. 27, 1204 (1971).

[5] P. D. Kim, I. A. Turpanov, S. V. Stolyar, R. S. Iskhakov, V. I. Yushkov, A. Y. Betenkova, L. A. Li, E. V. Bondareva, T. N. Isaeva, and M. M. Karpenko, Technical Physics 49, 431 (2004).

[6] J. A. Christodoulides, Y. Huang, Y. Zhang, G. C. Hadjipayanis, I. Panagiotopoulos, and D. Niarchos, J. Appl. Phys. 87, 6938 (2000).

[7] D. Halley, A. Marty, P. Bayle-Guillemaud, B. Gilles, J. P. Attane, and Y. Samson, Phys. Rev. B 70, 174438 (2004).

[8] O. Ersen, V. Parasote, V. Pierron-Bohnes, M. C. Cadeville, and C. Ulhaq-Bouillet, J. Appl. Phys. 93, 2987 (2003).

[9] T. A. Tyson, S. D. Conradson, R. F. C. Farrow, and B. A. Jones, Phys. Rev. B 54, R3702 (1996).

[10] M. Maret, C. Cadeville, R. Poinsot, A. Herr, E. Beaurepaire, and C. Monier, J. Magn. Magn. Mat. 166, 45 (1997).

[11] V. Gehanno, C. Revenant-Brizard, A. Marty, and B. Gilles, J. Appl. Phys. 84, 2316 (1998).

[12] A. Martins, M. C. A. Fantini, and A. D. Santos, J. Magn. Magn. Mat. 265, 13 (2003).

[13] L. G. Parrat, Phys. Rev. 95, 359 (1954).

[14] J. Als-Nielsen and D. McMorrow, Elements of Modern X-ray Physics (John Wiley and Sons, 2001).

[15] H. C. N. Tolentino, A. Y. Ramos, M. C. M. Alves, R. A. Barrea, E. Tamura, J. C. Cezar, and N. Watanabe, J. Synchrotron Rad. 8, 1040 (2001).

[16] A. L. Ankudinov, C. Bouldin, J. J. Rehr, J. Sims, and H. Hung, Phys. Rev. B 65, 104107 (2002).

[17] L. Seve, N. Jaouen, J. M. Tonnerre, D. Raoux, F. Bartolome, M. Arend, W. Felsch, A. Rogalev, J. Goulon, C. Gautier, et al., Phys. Rev. B 60, 9662 (1999).

[18] H. C. N. Tolentino, J. C. Cezar, N. Watanabe, C. Piamonteze, N. M. Souza-Neto, E. Tamura, A. Ramos, and R. Neueschwander, Physica Scripta 115, 977 (2005). 